$54^{\text {ème }}$ Congrès de la SFMBCB, 03001 (2011)

DOI: $10.1051 / \mathrm{sfmbcb} / 20115403001$

(C) Owned by the authors, published by EDP Sciences, 2011

\title{
Traitement de lésions papillomateuses multiples par injection intralésionnelle de Cidofovir chez un patient atteint du SIDA
}

\author{
Gaston $\mathbf{R}^{\mathbf{1}}$, Catros $\mathbf{S}^{\mathbf{1}}$, Cazanave $\mathbf{C}^{\mathbf{2}}$, Milpied $\mathbf{B}^{\mathbf{3}}$, Fricain $\mathbf{J C}^{\mathbf{1}}$ \\ ${ }^{1}$ Pôle d'Odontologie et de Santé buccale, Hôpital Pellegrin, CHU, Bordeaux, France \\ ${ }^{2}$ Service de Médecine interne, Hôpital Pellegrin, CHU, Bordeaux, France \\ ${ }^{3}$ Service de Dermatologie Hôpital St André, CHU, Bordeaux, France \\ jean-christophe.fricain@biophys.u-bordeaux2.fr
}

Le cidofovir (Vistide ${ }^{\circledR}$ ) ou (S)-1-[3-hydroxy (phosphonylméthoxypropyl)-cytosine] appartient à la famille des analogues nucléosidiques. Bien qu'initialement développés à des fins antitumorales, les analogues nucléosidiques possèdent une activité antivirale découverte dès la fin des années 70 (Descamps 1978). Le représentant le plus connu, l'aciclovir, est largement utilisé dans le traitement des infections herpétiques. Le cidofovir, analogue de la désoxycitidine monophosphate, possède une activité antivirale sur un large spectre de virus : herpès virus, polyomavirus, papillomavirus, adénovirus, poxvirus, cytomégalovirus (De Clercq et al. 1986). Il a obtenu une AMM en France pour sa forme injectable dans le traitement de la rétinite à cytomégalovirus chez le patient séropositif pour le VIH. Il a par ailleurs été utilisé avec succès en applications topiques dans les infections ano-génitales à HPV (Tyring 1997), ainsi qu'en injections intralésionnelles dans le traitement de la papillomatose laryngée récurrente (Donne 2008) et des papillomatoses nasales (Shemeno).

Le cas présenté concerne un patient suivi à la fin de l'année 2006 dans le Service d'Odontologie du CHU de Bordeaux pour le traitement de papillomes buccaux multiples évoluant depuis plusieurs mois. Le patient, âgé de 33 ans, présentait un SIDA déclaré et était hospitalisé dans le Service de Médecine interne pour la prise en charge d'une mycobactériose digestive atypique. La charge virale était de 40000 copies. $\mathrm{mL}^{-1}$ et le taux de CD4 de $61 . \mathrm{mm}^{-3}$. L'examen endobuccal révélait des lésions papillomateuses multiples, intéressant la muqueuse palatine, les joues et les lèvres, entraînant une gêne fonctionnelle. Devant l'aspect particulièrement végétant des lésions sur un terrain immunodéprimé, une biopsie a été réalisée afin d'éliminer une dégénérescence éventuelle. L'examen anatomopathologique a révélé une papillomatose avec une kératose de surface mais sans dysplasie. Initialement, un traitement topique par imiquimod (Aldara ${ }^{\circledR}$ ) et trétinoïne (Effederm ${ }^{\circledR}$ ) avait été instauré, mais il n'avait apporté aucune amélioration clinique après 2 mois d'utilisation. Une injection intra-lésionnelle de cidofovir $\left(5 \mathrm{~mL}\right.$ à la concentration de $\left.2,5 \mathrm{mg} \cdot \mathrm{mL}^{-1}\right)$ fut pratiquée en traitement d'attaque avec l'idée d'un relais par application topique de cidofovir en fonction du bénéfice clinique et des effets secondaires. Lors des consultations de contrôle réalisées une et deux semaines après l'injection, on observait une nette diminution des lésions labiales et palatines dans les zones injectées. Aucun effet secondaire local ou à distance n'a été mis en évidence. La poursuite du traitement par application locale de cidifovir n'a pas pu être réalisée en raison du décès du patient consécutif à l'aggravation de son infection digestive.

En conclusion, l'injection intralésionelle de cidofovir pourrait constituer un traitement de choix pour les lésions papillomateuses multiples chez le patient immunodéprimé. La voie locale se justifie par la possibilité d'obtenir in situ sur des lésions facilement accessibles, une importante concentration de principe actif, tout en limitant la néphrotoxicité du cidofovir (Chhetri 2002).Cependant, en raison de son coût important, l'usage du cidofovir dans cette indication doit être limité aux lésions invalidantes multiples et résistantes aux traitements classiques. 